\title{
The diagnosis and management of depression in the era of the DSM-5
}

\author{
Thomas E, 1,2*, MBchB, DipHIVMan, MMed(Psych), FCPsych; Seedat S, ${ }^{1,2}$, MBChB, FCPsych, MMed (Psych), PhD \\ ${ }^{1}$ South African Research Chairs Initiative (SARChl), PTSD program, Department of Psychiatry, Stellenbosch University, South Africa. \\ ${ }^{2}$ Department of Psychiatry, Stellenbosch University, Stellenbosch, South Africa \\ *Corresponding author, email: eileenthomas@sun.ac.za
}

\begin{abstract}
Depressive disorders comprise a range of conditions that can be viewed along many dimensions. The Diagnostic and Statistical Manual of Mental Disorders, 5th Edition (DSM-5) introduced several changes to the chapter on depressive disorders largely informed by advances in neuroscience, clinical and public health needs and difficulties identified with the previous DSM-IV classification system and criteria. Clinical characteristics vary across depressive disorders, as do the corresponding treatments. Pharmacotherapy and psychotherapy should be tailored to the individual patient.
\end{abstract}

Keywords: depression, depressive disorders, DSM-5, diagnosis, premenstrual dysphoric disorder

\section{Introduction}

According to the World Health Organization (WHO), depression is one of the most prevalent disorders, affecting an estimated $4.4 \%$ of the global population, and is the single largest contributor to disability worldwide (contributing $7.5 \%$ of all years lived with disability in 2015). ${ }^{1,2}$ More than $80 \%$ of this disease burden is carried by low- and middle- income countries (LMICs) with $16 \%$ of all cases of depressive disorders residing in Africa. ${ }^{3,4}$ In South Africa, the lifetime and 12-month prevalence rates for major depressive disorder are $9.7 \%$ and $4.9 \%$, respectively, with less than a quarter of South Africans with major depressive disorder able to access services and receive treatment. ${ }^{4}$ Depressive disorders are also a major contributor to death by suicide. In adults, depressive disorders increase in prevalence in the 20- to 30-year age group and continue to increase in middle age. These disorders are more common in individuals who are unmarried, divorced or widowed (compared to individuals who are married); in those who have suffered traumatic life events; and those who have a family history of depression. Women have a twofold greater risk of developing depression than men, with this gender differential manifesting at puberty. The course of depression is variable, both in terms of chronicity and remission. The probability of recurrence is also very high with approximately $80 \%$ of patients who achieve remission experiencing at least one recurrence in their lifetime. Psychiatric comorbidity (e.g. anxiety disorders, alcohol and drug-use disorders), history of childhood trauma, and greater severity of a depressive episode all predict a less favourable course. ${ }^{5}$

Reducing the incidence and prevalence of depression is a major public health priority. Similar to other LMICs, South Africa suffers from an absence of qualified mental health care workers. Only $0.9 \%$ of all registered psychiatrists in SA practise in primary health care settings. ${ }^{6}$ From this perspective, there is a key need to integrate the management of depression into general medical care, consistent with evidence from around the world highlighting the value of collaborative care. ${ }^{7}$ In view of the high prevalence of depression, escalating healthcare costs, shortage of mental health professionals, and high rates of medical comorbidity associated with depression (e.g. cardiovascular disease), uncomplicated depression should ideally be recognised and managed at primary care level. Family physicians and general practitioners therefore need to be well equipped to diagnose and treat depressive disorders.

The Diagnostic and Statistical Manual of Mental Disorders, 5th Edition (DSM-5) introduced several updates and changes to the chapter on depressive disorders. ${ }^{8}$ Changes to the DSM were largely informed by advances in neuroscience, clinical and public health needs and difficulties identified with the previous DSM-IV classification system and criteria. Much of the decision-making that informed the DSM-5 was also to ensure better alignment with the International Classification of Diseases and its upcoming 11 th edition (ICD-11). ${ }^{9}$

This review outlines some of the major changes including the introduction of two new disorders: disruptive mood dysregulation disorder (DMDD) and premenstrual dysphoric disorder (PMDD). We discuss a diagnostic approach, appropriate use of subtyping and specifiers, and lastly a comprehensive management plan that addresses these changes. 


\section{Diagnosis and identification}

Depression carries a wide differential diagnosis. If a depressive episode is diagnosed it is important to ascertain whether the depressive episode is part of a depressive disorder or another psychiatric or medical illness. MDD also needs to be differentiated from normal sadness or bereavement although in patients who are grieving, if depressive symptoms are severe and persistent, a diagnosis of MDD can be made. An accurate diagnosis can be determined through detailed history taking, mental status and physical examination, and supplementary laboratory tests as indicated.

\section{DSM-5 Depressive Disorders}

The DSM-5 depressive disorders include: disruptive mood dysregulation disorder, major depressive disorder (including major depressive episode), persistent depressive disorders (dysthymia), premenstrual dysphoric disorder, substance/ medication-induced depressive disorder, depressive disorder due to another medical condition, and other specified and unspecified depressive disorders. ${ }^{8}$

\section{Disruptive Mood Dysregulation Disorder (DMDD)}

This new disorder was included due to concerns that bipolar disorder was being overdiagnosed and treated in children. It refers to persistent irritability and frequent episodes of behavioural dyscontrol in children up to 12 years of age. ${ }^{10}$ Community surveys have estimated a prevalence of DMDD of $2-5 \%$ with a male preponderance. ${ }^{10}$

\section{Major Depressive Disorder}

As a syndrome, MDD is defined by a constellation of features that are related not only to mood but also to sleep, energy, cognition, motivation, and motor behavior persisting for $\geq 2$ weeks. The DSM-5 criteria for a major depressive episode are as follows:

Five or more out of 9 symptoms (including either depressed mood, or loss of interest or pleasure) in the same 2-week period. Each of these symptoms represents a change from previous functioning, and needs to be present nearly every day:

1. Depressed mood (subjective or observed); can be an irritable mood in children and adolescents, most of the day;

2. Loss of interest or pleasure, most of the day;

3. Change in weight or appetite. Weight: $5 \%$ change over 1 month;

4. Insomnia or hypersomnia;

5. Psychomotor retardation or agitation (observed);

6 . Loss of energy, or fatigue;

7. Worthlessness or guilt;

8. Impaired concentration or indecisiveness; or

9. Recurrent thoughts of death or suicidal ideation or attempt.

In the DSM-5 bereavement, formerly an exclusion criterion, no longer precludes making a diagnosis of MDD when syndromal criteria are otherwise fulfilled.

\section{Persistent Depressive Disorder (Dysthymia)}

DSM-5 has subsumed chronic major depression and dysthymia under the unified heading of persistent depressive disorder. Persistent depressive disorder (PDD) is chronic (depressive symptoms persist for more than 2 years). In addition to a pervasive depressed mood, only 2 of 6 symptoms are required for the diagnosis (sleep disturbance, appetite disturbance, loss of energy, decreased self-esteem, hopelessness, or poor concentration). If a patient meets criteria for MDD, then both diagnoses can be made (i.e. MDD and PDD).

\section{Premenstrual Dysphoric Disorder (PMDD)}

PMDD is a new category in DSM-5 that describes a constellation of affective, behavioural, cognitive and physical symptoms. A distinguishing characteristic of PMDD is the timing of symptom onset. In women with PMDD, mood symptoms occur only during the luteal phase of the menstrual cycle (ovulation until onset of menses) and resolve after menstruation onset. Women with PMDD report normal mood and functioning during the follicular phase of the menstrual cycle (first day of the menstrual cycle until ovulation). As such, PMDD consists of intermittently occurring symptoms, but is also characterised by mental state changes and impairment of functionality. PMDD is frequently missed, particularly as the clinical evaluation may not coincide with symptom occurrence, or there may be clinician unawareness or failure of patients to recognise that intermittent symptoms are treatable. The diagnosis is made when a patient has at least 5 of 11 specific symptoms (marked depressed mood, marked anxiety, marked affective lability [e.g. feeling suddenly sad or fearful, or heightened sensitivity to rejection], persistent or marked anger or irritability, sleep disturbances, appetite disturbances, decreased energy or lethargy, concentration problems, decreased interest, feeling overwhelmed or out of control, and other physical symptoms [e.g. breast tenderness, headache, bloating]) that occur during the week before menses onset, and improve within a few days after the onset of menses.The symptoms are severe enough to interfere significantly with social, occupational, scholastic or sexual functioning. A systematic approach to the diagnosis of PMDD is essential and should include ruling out a premenstrual exacerbation of another underlying or comorbid mood, anxiety or physical disorder. ${ }^{11,12}$.

\section{Substance/Medication-induced Depressive Disorder}

This diagnosis can be made when depressive symptoms appear during or within one month after using a substance and there is confirmatory evidence from the patient's history, physical examination, or laboratory findings of substance use, abuse, intoxication, or withdrawal prior to the start of the depressive symptoms.

\section{Depressive Disorder due to another medical condition}

To arrive at a primary psychiatric diagnosis, all potential secondary causes must be ruled out. This includes systematic screening for possible drug-induced psychopathology related not only to drugs of abuse, but also to prescription medications, some of which can have serious iatrogenic effects, including 
depression. The other important cause to rule out is the possibility of a general medical condition triggering psychiatric symptoms, which requires targeted questioning about medical history, a review of organ systems, and ordering key laboratory tests. In the DSM-5, depressive episodes that arise secondary to a medical condition (e.g. hypothyroidism), substance abuse (e.g. alcohol), or iatrogenically from medications (e.g. corticosteroids, oral contraceptives) are viewed as distinct from MDD in regard to risk of recurrence, and neurobiological underpinnings.

\section{Other Specified and Unspecified Depressive Disorder}

Depressive states that involve 2 to 4 associated symptoms lasting $\geq 2$ weeks but $\leq 2$ years are sometimes described as minor depression, captured in the DSM-5 as "other specified and unspecified depressive disorder". This category may wrongly infer low severity and therefore discourage treatment.

\section{Case example}

Mrs S, aged 35, presents with depressive symptoms that began 6 weeks ago. Symptoms include: depressed mood, hopelessness, anhedonia, anxiety and insomnia. She denies suicidal ideation. She had one previous depressive episode following the birth of her second child 2 years prior. The antidepressant sertraline (100 mg per day) successfully alleviated her depressive symptoms. She has no medical or substance use history of note. A mental state examination reveals low mood, tearfulness, coherent thought form and no perceptual disturbances. Her score on assessment with the Hamilton Depression Rating Scale (HAM-D) is $20 / 30$. Physical examination is unremarkable.

Once a diagnosis of MDD is made, Mrs $\mathrm{S}^{\prime}$ condition can be further characterised using specifiers. The first specifier is the severity of MDD which needs to be rated as mild, moderate or severe. The severity of functional impairment and/or suicidality and/or the presence of psychotic symptoms are important indicators of the severity of a depressive episode.

\section{Other specifiers include:}

\section{With anxious distress}

This specifier refers to prominent anxiety features (feeling worried, restless, tense or keyed up; fearful of losing control or something terrible happening). Co-occurring anxiety is prevalent in patients with MDD, causes impairment and/or distress and is associated with the reporting of suicidal thoughts. Anxiety symptoms or syndromes occur in at least half of outpatients who have MDD and can account for a substantial percentage of nonresponse to first-line antidepressant therapy. ${ }^{13}$

\section{With mixed features}

MDD lies on a continuum with bipolar disorder. Patients with either diagnosis can manifest some features of the other. For example, some patients with MDD may have racing thoughts and a reduced need for sleep. At least 3 symptoms of mania or hypomania (elevated or expansive mood, heightened selfesteem or grandiosity, racing thoughts, increased speech or pressure of speech, increased energy, excessive involvement in behaviour with a potential for negative consequences, and/or reduced need for sleep) need to be present. The mixed feature specifier can be applied to depressed patients whether or not they have ever had a manic or hypomanic episode in the past, MDD rather than bipolar disorder remains the overarching diagnosis, unless criteria have ever been met for a full mania or hypomania in which case the diagnosis of Bipolar Disorder rather than MDD needs to be made.

Patients with bipolar depression may be erroneously diagnosed initially and treated for Major Depressive Disorder ${ }^{14}$ and treated with antidepressant monotherapy, which is not efficacious as stand-alone pharmacotherapy and may be associated with mood switching. ${ }^{15}$ As depression is frequently the presenting mood state, screening for previous episodes of hypomania/mania is essential. Several additional cues may point to presence of a bipolar depression such as early onset, family history of bipolar disorder, treatment resistance, number of previous episodes, and rapid response to antidepressant therapy. ${ }^{16}$

\section{With melancholic features}

Melancholia involves the cardinal symptoms of anhedonia and lack of mood reactivity, alongside features such as distinct quality of mood, diurnal variation, excessive guilt, and sever weight loss. Melancholic features do not necessarily recur across successive episodes of depression. However, melancholic features carry an increased risk of psychosis and high-lethality suicidal behaviour.

\section{With atypical features}

This comprises a symptom constellation of:

- Reactiveness of mood to environmental stimuli (unlike melancholia).

- Heightened interpersonal sensitivity

- Hypersomnia

- Hyperphagia

- Profound fatigue or a sense of physical heaviness

\section{With mood-congruent/ incongruent psychotic features}

Psychotic symptoms in MDD are mainly mood-congruent. This means that the content of the delusions and hallucinations are consistent with the typical themes of depression, such as guilt, personal inadequacy, death, disease, and nihilism. Sometimes, delusions and hallucinations do not include these depressive themes (i.e. are mood-incongruent). The presence of psychosis and related components (e.g. mood congruence) is closely linked with the severity of depression (high severity) and poorer prognosis with implications for treatment.

\section{With Catatonia}

The DSM-5 now allows for specification of catatonia in a variety of disorders, including depression. ${ }^{8}$ In previous editions catatonia was associated with schizophrenia and listed as a subtype. Catatonia refers to psychomotor disturbances. The DSM-5 criteria include the presence of three symptoms from the following list of twelve: stupor (i.e. no psychomotor activity; not actively relating to environment); catalepsy 
(i.e. passive induction of a posture held against gravity); waxy flexibility (i.e. allow positioning by examiner and maintain position); mutism; negativism (i.e. opposition or no response to instructions or external stimuli); posturing (i.e. spontaneous and active maintenance of a posture against gravity); mannerisms (i.e. odd, circumstantial caricature of normal actions; stereotypy (i.e. repetitive, abnormally frequent, non-goal-directed movements); agitation; grimacing; echolalia; and echopraxia. Other common symptoms are motor resistance to simple commands, posturing, rigidity, automatic obedience, and repetitive movements.

\section{With peripartum onset}

The DSM-5 does not recognise postpartum depression as a separate diagnosis; rather, a patient must meet the criteria for a major depressive episode and the criteria for the peripartumonset specifier. The definition is therefore a major depressive episode with an onset in pregnancy or within 4 weeks of delivery. The clinical rationale for including this is to remind clinicians of the treatment implications associated with the occurrence of MDD in this reproductive period. About half of patients identified with postpartum depression will already report an onset of symptoms during pregnancy.

\section{With seasonal pattern}

DSM-5 identifies "with seasonal pattern" as a specifier for recurrent major depression. This is when depressive episodes occur in line with seasonal changes. A winter-type seasonal pattern is most common, especially in higher latitudes, with a summer-type seasonal pattern less often diagnosed.

\section{Severity}

This provides important details about the presentation and is informative for promoting more appropriate treatment. Mild major depression can be treated with psychotherapy alone while moderate to severe episodes should be treated with medication or with a combination of medication and psychotherapy. It is important to take patient preference, prior treatment history and affordability into account. Moderate-severe and severe depression also requires that decisions about treatment setting (inpatient or outpatient) and other therapeutic modalities be considered (e.g. electroconvulsive therapy which is more appropriate than psychotherapy for catatonic depression).

\section{Suicide risk assessment}

As part of the clinical evaluation, a comprehensive risk assessment needs to be conducted and documented. Evaluate the patient for risk and protective factors. Key risk factors include previous suicide attempts, a family history of suicide, access to lethal means, history of a psychiatric disorder, history of alcohol and substance abuse, and severe hopelessness. ${ }^{17}$

\section{Rating scales and screening tools}

There are several brief, useful and easy-to-administer ratingscales which can be used to guide diagnosis and monitor care, and are easily incorporated into clinical practice. This information can be used to follow the progression of symptoms and effectiveness of treatment. Although rating scales should never be used alone to establish a diagnosis or treatment plan, they can and should be used to augment information from the clinician's diagnostic assessment and follow-up interviews.

\section{Patient Health Questionnaire (PHQ-9)}

The PHQ-9 is a 9-item self-report questionnaire that can assist in the detection of depression and supplement a thorough psychiatric and mental health status assessment. The PHQ-9 is available online at no cost and in several languages and takes few minutes to complete. ${ }^{18}$

\section{Edinburgh Postnatal Depression Scale (EDPS)}

This 10-item self-report measure is designed to screen women for symptoms of emotional distress during pregnancy and the postnatal period. ${ }^{19}$

\section{Daily Rating of Severity of Problems (DRSPP)}

A diagnosis of PMDD requires prospective daily monitoring of symptoms for $\geq 2$ consecutive months. The widely used DRSPP allows clinicians to quantify the severity of physical, emotional, and behavioral symptoms, is easy to use in clinical practice, and creates a graphic representation of cyclical symptom changes. The DRSPP includes all PMDD symptoms and severity ratings and is recognised as a valid instrument for diagnosing PMDD. ${ }^{11}$

\section{Columbia Suicide Severity Rating Scale (C-SSRS)}

The C-SSRS is a 2-page questionnaire that only takes a few minutes to administer and can also be completed as a selfreport measure. It can be used to assess severity of and track changes over times in suicidal ideation and behaviour. The "Lifetime/Recent" version allows clinicians to gather lifetime history of suicidality as well as any recent suicidal ideation and/ or behaviour, whereas the "since last visit" version assesses suicidality in patients who have completed at least 1 Lifetime/ Recent C-CSSRS assessment. ${ }^{20}$

\section{Collaborative Assessment and Management of Suicidality (CAMS)}

The CAMS is a structured, evidence-based method of risk assessment and treatment planning. The CAMS provides a framework to involve the patient in the assessment of their suicidality and to design a suicide-specific treatment plan. ${ }^{21}$.

\section{Management}

\section{General principles}

- Establish a therapeutic alliance; simply seeking psychiatric care can bring relief by providing some sense of control over distressing symptoms. Validate the patient's experience, normalise their response and instill a sense of hope. Be nonjudgmental and empathic as this will help the patient accept his/her experiences and emotions. Engage the patient as an active participant in their treatment plan.

- Treat comorbid illnesses, being mindful of both diseasedisease, disease-drug and drug-drug interactions. For example, depression and cardiovascular disease are often comorbid, 
and require avoidance of tricyclic antidepressants (TCAs) as TCAs may exacerbate cardiac conduction abnormalities. Drug-drug interactions are less likely if a medication has a wide therapeutic index, is a "weak" inducer or inhibitor of metabolism and the patient has normal renal and hepatic function.

- Treatment should be guided by subtype as well as severity of the depressive disorder (e.g. the management of secondary depression involves addressing the underlying medical condition and might include use of antidepressant medication).

- Pay careful attention to suicidal ideation and suicidality, including during antidepressant therapy.

- Coordinate treatment with a psychiatrist and other specialists as needed.

\section{Non-pharmacological}

\section{- Lifestyle modification}

It is important to educate patients about adopting a healthy lifestyle; eating wisely, not smoking, sleep hygiene and engaging in regular aerobic exercise. Mood episodes have been shown to be associated with significant elevations in inflammatory cytokines in serum and cerebrospinal fluid (CSF) including interleukins, tumor necrosis factor-alpha, interferon gamma and C-reactive protein. A sedentary lifestyle increases depression risk, whereas regular exercise has been shown to down-regulate systemic inflammation. A Mediterranean diet which comprises of fruits, vegetables, legumes and food rich in omega-3 fatty acids (fish, nuts, leafy green vegetables) has antiinflammatory effects. ${ }^{22}$ Lycopene-containing foods (tomatoes, papaya, carrots, watermelon) are rich in antioxidants and thus reduce inflammation. Obesity is a pro-inflammatory state, and when an obese person loses weight, levels of inflammatory markers (interleukin-6, leptin) decrease. Neuroinflammation is a well-established underlying mechanism in depression. ${ }^{23}$ Encourage the patient to adopt an exercise regimen that is appropriate and can be maintained by the patient.

- Psychotherapy: in addition to developing and sustaining a therapeutic alliance, psychotherapy by a trained professional can be transformational by helping patients to process complex life events and emotions, feel understood, enhance self-esteem and overcome psychological barriers to recovery. Evidence based psychotherapies (e.g. cognitive behavioural therapy (CBT) and interpersonal therapy), as adjuncts to pharmacotherapy can further improve outcomes in MDD. ${ }^{24}$

- Refer patient to local support groups such as the South African Depression and Anxiety group (SADAG).

- Phototherapy remains a standard treatment for MDD with a seasonal pattern, but lacks demonstrated efficacy in nonseasonal forms of depression. ${ }^{25}$

\section{Pharmacological}

- Depression is associated with multiple genetic and environmental causes, hence it is not surprising that a myriad of pharmacological treatments can be used. When deciding on the best agent, clinicians should adopt an individualised, tiered approach that is guided by, inter alia, symptoms that are most prominent, comorbid illness, patient preference, medication availability and cost. First-choice agents are selective serotonin reuptake inhibitors (SSRIs) and serotonin norepinephrine reuptake inhibitors (SNRIs).

- Renal disease can play a significant role in altering the pharmacokinetics of a medication, especially in elimination, clearance and plasma protein binding. Dosage adjustment and caution are needed when using a psychotropic as severity of renal impairment increases. The pharmacokinetics of fluoxetine remain unchanged irrespective of the stage of renal impairment. Patients in active renal dialysis report good tolerability and efficacy.

- Consider 'symptom targeted, 2 birds with one stone-approach'. Consider comorbid disease states that allow for prescribing 1 medication to treat 2 conditions, thereby reducing pill burden (e.g. duloxetine for comorbid depression and diabetic neuropathy). In patients who report weight loss and poor sleep, a sedating antidepressant such as Mirtazapine may be a good option ( $\mathrm{H} 1$ receptor antagonism dampens satiety response, resulting in increased caloric intake).

- For PMDD,antidepressants have been shown to effectively ameliorate affective and physical symptoms and improve quality of life and psychosocial function in patients with PMD. ${ }^{26}$ However, only antidepressants that affect serotonergic - not noradrenergic - transmission are effective in PMDD. These include: the tricyclic antidepressant clomipramine, the SSRIs citalopram, escitalopram, fluoxetine, paroxetine, and sertraline and the serotonin-noradrenergic reuptake inhibitor venlafaxine.

- Anxious depression: Serotonergic antidepressants remain the mainstay of treatment for depression with anxiety.

- Melancholic depression requires antidepressant treatment or ECT rather than psychotherapy. Research indicates that TCS might yield better results than SSRIs

- While there are no FDA-approved drugs for treating persistent depressive disorder (dysthymia), SSRIs and SNRIs are first-line pharmacological agents.

- Avoid polypharmacy, but also do not undertreat. Start with 1 medication, assess its efficacy, tolerability and safety before adding an adjunct. SSRIs and SNRIs are associated with a delayed response to treatment (several weeks).

- Regular review is crucial not only for treatment response but also for monitoring the emergence of any side-effects. Gradual titration can circumvent adverse effects and help establish lowest effective dose.

- The continuation period of treatment is dependent on whether the depression is a first or recurrent episode. For a first episode, a minimum period of 6-9 months of maintenance is required. For a recurrent episode, the recommended minimum treatment length is 3 years. 


\section{Conclusion}

In summary, depressive disorders are common and associated with increased psychosocial morbidity and mortality. Early identification and tailored treatment may reduce the impact.

\section{Acknowledgements}

Nil

\section{Funding support}

This work is supported by the South African Research Chair in PTSD hosted by Stellenbosch University, funded by the DST and administered by NRF and the South African Medical Research Council.

\section{Conflict of interest and funding}

There is no conflict of interest in the present review for any of the authors.

\section{References}

1. Depression and Other Common Mental Disorders Global Health Estimates. Accessed on 4 Feb 2018. Available from: http://apps.who.int/iris/ bitstream/10665/254610/1/WHO-MSD-MER-2017.2-eng.pdf

2. Whiteford HA, Degenhardt $L$, Rehm J, et al. Global burden of disease attributable to mental and substance use disorders: findings from the Global Burden of Disease Study 2010. Lancet. 2013;382(9904):1575-86. doi:10.1016/ S0140-6736(13)61611-6

3. Seedat S, Scott KM, Angermeyer MC, et al. Cross-National Associations Between Gender and Mental Disorders in the World Health Organization World Mental Health Surveys. Arch Gen Psychiatry. 2009;66(7):785. doi:10.1001/ archgenpsychiatry.2009.36

4. Herman AA, Stein DJ, Seedat S, Heeringa SG, Moomal H, Williams DR. The South African Stress and Health (SASH) study: 12-month and lifetime prevalence of common mental disorders. S Afr Med J. 2009;99(5 Pt 2):339-44.

5. Otte C, Gold SM, Penninx BW, et al. Major depressive disorder. Nat Rev Dis Prim. 2016;2:16065. doi:10.1038/nrdp.2016.65

6. De Kock JH, Pillay BJ. A situation analysis of psychiatrists in South Africa's rural primary healthcare settings. Afr J Prim Health Care Fam Med. 2017;9(1):e1-e6. doi:10.4102/PHCFM.V911.1335

7. Raney LE. Integrating Primary Care and Behavioral Health: The Role of the Psychiatrist in the Collaborative Care Model. Am J Psychiatry. 2015;172(8):721-8. doi:10.1176/appi.ajp.2015.15010017

8. Diagnostic and Statistical Manual of Mental Disorders Fifth Edition. 5th ed. Arlington, VA: American Psychiatric Publishing; 2013.

9. Regier DA, Kuhl EA, Kupfer DJ. The DSM-5: Classification and criteria changes. World Psychiatry. 2013;12(2):92-8. doi:10.1002/wps.20050

10. Copeland WE, Angold A, Costello EJ, Egger H. Prevalence, comorbidity, and correlates of DSM-5 proposed disruptive mood dysregulation disorder. Am J Psychiatry. 2013;170(2):173-9. doi:10.1176/appi.ajp.2012.12010132
11. Pearlstein T. Treatment of Premenstrual Dysphoric Disorder: Therapeutic Challenges. Expert Rev Clin Pharmacol. Feb 2016:1-4. doi:10.1586/17512433.20 16.1142371

12. Robinson LL, Ismail KM. Clinical epidemiology of premenstrual disorder: informing optimized patient outcomes. Int J Womens Health. 2015;7:811-8. doi:10.2147/JJWH.S48426

13. Hirschfeld RMA. The Comorbidity of Major Depression and Anxiety Disorders: Recognition and Management in Primary Care. Prim Care Companion J Clin Psychiatry. 2001 [Accessed on 27 Feb 2018];3(6):244-54. Available from: http:// www.ncbi.nlm.nih.gov/pubmed/15014592

14. Smith DJ, Griffiths E, Kelly M, Hood K, Craddock N, Simpson SA. Unrecognised bipolar disorder in primary care patients with depression. $\mathrm{Br} J$ Psychiatry. 2011;199(1):49-56. doi:10.1192/bjp.bp.110.083840

15. Baldessarini RJ, Tondo L, Visioli C. First-episode types in bipolar disorder: predictive associations with later illness. Acta Psychiatr Scand. 2014;129(5):38392. doi:10.1111/acps.12204

16. Swann AC, Geller B, Post RM, et al. Practical Clues to Early Recognition of Bipolar Disorder: A Primary Care Approach. Prim Care Companion J Clin Psychiatry. 2005 [Accessed 26 Feb 2018];7(1):15-21. Available from: http://www.ncbi.nlm.nih.gov/ pubmed/15841189

17. Franklin JC, Ribeiro JD, Fox KR, et al. Risk Factors for Suicidal Thoughts and Behaviors: A Meta-Analysis of 50 Years of Research. doi:10.1037/bul0000084

18. Arroll B, Goodyear-Smith F, Crengle S, et al. Validation of PHQ-2 and PHQ-9 to screen for major depression in the primary care population. Ann Fam Med. 8(4):348-53. doi:10.1370/afm.1139

19. Boyd RC, Le HN, Somberg R. Review of screening instruments for postpartum depression. Arch Womens Ment Health. 2005;8(3):141-53. doi:10.1007/ s00737-005-0096-6

20. John Mann by, Oquendo M, Posner K. COLUMBIA-SUICIDE SEVERITY RATING SCALE (C-SSRS). Clin Pract. 2003:103-30. Accessed on 26 Feb 2018. Available from: http://cssrs.columbia.edu/wp-content/uploads/C-SSRS_PediatricSLC_11.14.16.pdf

21. Jobes DA, Wong SA, Conrad AK, Drozd JF, Neal-Walden T. The Collaborative Assessment and Management of Suicidality versus Treatment as Usual: A Retrospective Study with Suicidal Outpatients. 483 Suicide Life-Threatening Behav. 2005 [Accessed on 30 Jan 2018];35(5). Available from: https://msrc.fsu. edu/system/files/A collaborative assessment and management of suicidality method shows effect.pdf

22. Esposito K, Di Palo C, Maiorino Ml, et al. Long-Term Effect of MediterraneanStyle Diet and Calorie Restriction on Biomarkers of Longevity and Oxidative Stress in Overweight Men. Cardiol Res Pract. Jan 2011:1-5. Available from: http://10.0.15.221/2011/293916

23. Chabry J, Nicolas S, Cazareth J, et al. Enriched environment decreases microglia and brain macrophages inflammatory phenotypes through adiponectindependent mechanisms: Relevance to depressive-like behavior. Brain, Behav Immun. 2015;50:275-87. Available from: http://10.0.3.248/j.bbi.2015.07.018

24. Hofmann SG, Asnaani A, Vonk IJJ, Sawyer AT, Fang A. The Efficacy of Cognitive Behavioral Therapy: A Review of Meta-analyses. Cognit Ther Res. 2012;36(5):42740. doi:10.1007/s10608-012-9476-1

25. Thalén B-E, Kjellman BF, Mørkrid L, Wibom R, Wetterberg L. Light treatment in seasonal and nonseasonal depression. Acta Psychiatr Scand. 1995;91(5):352-60. doi:10.1111/j.1600-0447.1995.tb09794.x

26. Marjoribanks J, Brown J, O'Brien PMS, Wyatt K. Selective serotonin reuptake inhibitors for premenstrual syndrome. Cochrane Database Syst Rev. June 2013. doi:10.1002/14651858.CD001396.pub3 